\title{
The Clinical Value of NLR, D-D and CRP/ALB Ratio in the Diagnosis of Pulmonary Thromboembolism
}

\author{
Tiantian Shan*, Zhen Cheng*, Min Yan, Xiangtao Pan\#" \\ Department of Hematology, Taicang Hospital Affiliated to Soochow University, Taicang, China \\ Email: "panxiangtao@csco.ac.cn
}

How to cite this paper: Shan, T.T., Cheng, Z., Yan, M. and Pan, X.T. (2021) The Clinical Value of NLR, D-D and CRP/ALB Ratio in the Diagnosis of Pulmonary Thromboembolism. Journal of Biosciences and Medicines, 9, 65-73.

https://doi.org/10.4236/jbm.2021.910006

Received: September 9, 2021

Accepted: October 15, 2021

Published: October 18, 2021

Copyright $\odot 2021$ by author(s) and Scientific Research Publishing Inc. This work is licensed under the Creative Commons Attribution International License (CC BY 4.0).

http://creativecommons.org/licenses/by/4.0/

\begin{abstract}
Objective: To evaluate the clinical diagnostic value of C-reactive protein/albumin ratio (CAR), neutrophil/lymphocyte ratio (NLR) and D-dimer (D-D) in patients with pulmonary thromboembolism (PTE). Methods: We conducted a retrospective analysis comparing hematology and coagulation in 362 PTCA-confirmed PTE patients with the control group and analyzing their relationships with CAR, NLR, and D-D. Receiver operating characteristic curve (ROC) was used to analyze the diagnostic threshold, area under the curve (AUC), diagnostic sensitivity and specificity of CAR, NLR and D-D for PTE. Results: 1) CAR, NLR and D-D levels in PTE patients were $2.13 \pm 2.08$, $8.96 \pm 1.94$ and $9.69 \pm 8.61$ respectively, significantly higher than those in control group $(\mathrm{CAR}=0.03 \pm 0.01, \mathrm{t}=20.7736, P<0.01 ; \mathrm{NLR}=1.76 \pm 0.53, \mathrm{t}$ $=2.4281, P<0.05$ and $\mathrm{PTE}=0.20 \pm 0.11, \mathrm{t}=3.0066, P<0.01$ respectively). 2$)$ NLR was positively correlated with CAR $(\mathrm{r}=0.2111, \mathrm{t}=4.0971, P<0.01)$ and $\mathrm{D}-\mathrm{D}(\mathrm{r}=0.1065, \mathrm{t}=2.0481, P<0.05)$, but CAR was not correlated with $\mathrm{D}-\mathrm{D}$ $(\mathrm{r}=0.0975, P>0.05) .3)$ The levels of HB, LY, PLT and AT in PTE patients were significantly lower than those in control group, while WBC, NE and FB were significantly higher than those in control group. 4) CAR was negatively correlated with $\mathrm{Hb}$ and AT $(P$ all $<0.01)$, but positively correlated with $\mathrm{WBC}$, NE, MO and FB $(P$ all $<0.01)$. NLR was negatively correlated with LY and AT $(P$ all $<0.01)$, but positively correlated with WBC, NE and FB $(P$ all $<0.01)$. DD was negatively correlated with $\mathrm{Hb}$ and PLT $(P$ all $<0.05)$, but positively correlated with WBC, NE and MO $(P$ all $<0.01)$. Conclusion: The levels of $\mathrm{Hb}, \mathrm{LY}, \mathrm{PLT}$ and AT were significantly decreased in PTE patients, while WBC, NE and FB were significantly increased. CAR, NLR and D-D were highly expressed in PTE patients, and were closely correlated with Hb, AT
\end{abstract}


and FB. Combined detection of CAR, NLR and D-D can improve the diagnostic value of PTE.

\section{Keywords}

Pulmonary Thromboembolism, CAR, C-Reactive Protein/Albumin Ratio, Neutrophil to Lymphocyte Ratio, Plasma D-Dimer, Clinical Diagnosis

\section{Introduction}

PTE which is a disease that seriously affects the life of patients shows a trend of increasing. PTE is the most common type of pulmonary embolism, and the thrombosis that causes PTE mainly comes from the formation of deep vein thrombosis (DVT), so DVT is the main cause of pulmonary embolism. PTE and DVT have the same triggers, including any factors that can lead to venous blood flow stasis, endothelial damage, and hypercoagulable stage. Recent studies have shown some connections between PTE and inflammatory response [1]. Inflammation can be both a cause as well as consequence of PTE [2]. Several components of the immune system are all involved in underlying inflammatory processes of PTE [2]. Neutrophil (NE) to lymphocyte (LY) ratio (NE/LY, NLR) is an important marker which can reflect the state of inflammation and immune function of the body. Neutrophils increase and/or lymphocytes decrease during inflammatory response [3]. However, recent studies [4] [5] have found that NLR is closely related to the occurrence and development of thrombotic diseases. C-reactive protein (CRP) is also an important marker for inflammation. Recent studies [6] [7] [8] have found that the ratio of CRP to nutritional index albumin (ALB) (CRP/ALB, CAR) is significant in judging the prognosis of malignant tumor, but there are few researches in thrombotic diseases. No studies have been reported on the clinical diagnostic value of combined detection of NLR, D-D and CAR for PTE. Therefore, this study retrospectively analyzed various indexes of hematology and coagulation in 362 patients with PTE, analyzed and discussed the diagnostic significance of combined detection of NLR, D-D and CAR for PTE, providing theoretical and experimental basis for better clinical diagnosis and treatment.

\section{Materials and Methods}

\subsection{Materials}

From January 1, 2015 to December 31, 2019, there were 270,983 discharged patients in our hospital, and 362 PTE patients confirmed by CTPA and other methods with complete data and clear prognostic records, including 214 males and 148 females, aged from 18 to 93, with an average age of 71.2 years old. Tumor embolism, amniotic fluid embolism, fat embolism and other non-venous thromboembolism were excluded. The medical record number, name, gender, 
age, inpatient department, primary disease(s), inducing factor(s), occurrence location(s), preventive measure(s), relevant examination result(s), treatment method(s) and prognosis of all cases were collected. 32 healthy subjects were selected as the control group, including 19 males and 13 females, aged from 29 to 51.

This study was discussed and approved by the Ethics Committee of Taicang Hospital affiliated to Soochow University, and informed consent was signed by relevant personnel.

\subsection{Methods}

The data of hospitalized VTE cases discharged from our hospital from January 1, 2015 to December 31, 2019 were provided by the HOSPITAL'S VTE network, and all relevant medical records were retrieved through the hospital's medical record center, including Ultrasonography or CT pulmonary angiogram (CTPA), D-D, CRP, prothrombin time (PT), thrombin time (TT), antithrombin III (AT-III, $\mathrm{AT}$ ), fibrinogen (FB) and International Normalized Ratio (INR), as well as hemoglobin (HB), white blood cell (WBC), NE, LY, monocyte (MO) and platelet (PLT) counts. A total of 362 cases of PTE with definite diagnostic basis and prognosis were included in this study.

\subsection{Statistical Analysis}

SPSS 23.0 software was used for statistical analysis. T-test was used for comparison of measurement data of two groups, Chi-square test for comparison of counting date, and Person correlation analysis for correlation analysis. The receiver operator characteristic curve (ROC) was used to analyze the diagnostic threshold, areas under the curve (AUC), diagnostic sensitivity and specificity of NLR, D-D and CAR for PTE. $P<0.05$ was considered as statistically significant.

\section{Results}

\subsection{Results of D-D, NLR, CAR and Other Inflammatory Indicators in PTE Patients and Control Group}

Comparison results of NLR and other inflammatory indicators between 362 PTE patients and the control group are shown in Table 1. As shown in Table 1, the differences of NLR, LY/MO ratio (LMR), CRP, ALB, CAR, PLT/LY ratio (PLR) and D-D between PTE patients and the control group were statistically significant. Considering that CRP and ALB had constituted a new indicator CAR, therefore, we selected NLR, LMR, CAR, PLR and D-D as research parameters for correlation analysis. Specific results are shown in Table 2.

Table 2 shows that the degree of correlation among NLR, LMR and PLR was the highest. The main reason for the result was that there were repeated parameters in these three indicators. In order to avoid this impact, NLR, D-D and CAR which had no repeated parameters were selected in this study as the indicators for judging diagnostic value of PTE patients. 
Table 1. Comparison results of NLR, D-D, CAR and other inflammatory indicators between PTE patients and control group.

\begin{tabular}{cccc}
\hline Inflammatory biomarkers & PTE group $(n=362)$ & Normal control group $(n=32)$ & $t$ \\
\hline NLR & $8.96 \pm 1.94$ & $1.76 \pm 0.53$ & $2.4281^{\star}$ \\
LMR & $2.52 \pm 1.91$ & $6.96 \pm 2.32$ & $9.0409^{* *}$ \\
CRP & $67.30 \pm 54.92$ & $1.00 \pm 0.01$ & $3.1189^{* *}$ \\
ALB & $34.56 \pm 5.16$ & $38.53 \pm 6.16$ & $2.4963^{*}$ \\
CAR & $2.13 \pm 2.08$ & $0.03 \pm 0.01$ & $20.7736^{* *}$ \\
SII & $1750.56 \pm 762.77$ & $403.65 \pm 153.25$ & 1.9486 \\
PLR & $230.29 \pm 198.53$ & $127.39 \pm 29.46$ & $2.0775^{\star}$ \\
D-D & $9.69 \pm 8.61$ & $0.20 \pm 0.11$ & $3.0066^{* *}$ \\
\hline${ }^{*} P<0.05,{ }^{* *} P<0.01$, the rest are all $>0.05 . \mathbf{\Lambda}:$ SII, Systemic immune inflammatory index, rank sum test $P=$ \\
0.000.
\end{tabular}

Table 2. Correlation between inflammatory markers NLR, CAR, D-D, PLR and LMR.

\begin{tabular}{cccccc}
\hline Comparative items & $r$ & $t$ & Comparative items & $R$ & $t$ \\
\hline NLR/D-D & 0.1065 & $2.0481^{*}$ & DD/CAR & 0.0975 & 1.8609 \\
NLR/CAR & 0.2111 & $4.0971^{* *}$ & DD/PLR & -0.0020 & 0.0378 \\
NLR/PLR & -0.7252 & $20.0886^{* *}$ & DD/LMR & -0.1215 & $2.3231^{\star}$ \\
NLR/LMR & -0.3068 & $6.1116^{* *}$ & CAR/PLR & 0.1256 & $2.4015^{*}$ \\
LMR/PLR & -0.2613 & $5.1336^{* *}$ & CAR/LMR & -0.1994 & $3.8585^{* *}$ \\
\hline
\end{tabular}

${ }^{\star} P<0.05,{ }^{*} P<0.01$, the rest are all $>0.05$.

\subsection{Comparison of Hematology and Coagulation Indexes between PTE Patients and Control Group}

The comparison results of hematology and coagulation indexes between the two groups are shown in Table 3. The results of correlation analysis between NLR, D-D, CAR and hematology, coagulation indicators are shown in Table 4. In addition, 90 patients had lower AT level than normal (24.9\%), and 97 patients had higher FB level (26.8\%).

\subsection{Comparison of Diagnostic Value of NLR, D-D, and CAR Respectively and in Combination for PTE}

The comparison results are shown in Table 5 and Figure 1. It can be seen from Figure 1 and Table 5 that although the detection of NLR, D-D and CAR respectively were of certain significance, the combined detection of them was of greater value.

\section{Discussion}

The importance of D-D in thrombotic diseases has been widely recognized, and it is related to pulmonary thromboembolism, acute myocardial infarction, cerebral infarction and other thrombotic diseases [9]. D-D in this study was significantly 
Table 3. Comparison of hematology and coagulation indexes between PTE patients and control group.

\begin{tabular}{|c|c|c|c|c|}
\hline Items & $\begin{array}{l}\text { Comparative } \\
\text { indicators }\end{array}$ & $\begin{array}{l}\text { PTE group } \\
(n=362)\end{array}$ & $\begin{array}{c}\text { Control group } \\
\quad(n=32)\end{array}$ & $t$ \\
\hline \multirow{6}{*}{$\begin{array}{l}\text { 1) Comparison } \\
\text { of hematology } \\
\text { indicators }\end{array}$} & $\mathrm{Hb}(\mathrm{g} / \mathrm{L})$ & $120.33 \pm 23.71$ & $139.20 \pm 12.48$ & $4.4665^{\star \star}$ \\
\hline & $\mathrm{WBC}\left(\times 10^{9} / \mathrm{L}\right)$ & $8.70 \pm 5.69$ & $5.68 \pm 1.51$ & $2.9860^{\star *}$ \\
\hline & $\mathrm{NE}\left(\times 10^{9} / \mathrm{L}\right)$ & $6.83 \pm 5.13$ & $3.25 \pm 1.15$ & $3.9362^{\star *}$ \\
\hline & $\mathrm{LY}\left(\times 10^{9} / \mathrm{L}\right)$ & $1.11 \pm 0.59$ & $1.94 \pm 0.45$ & $7.8598^{\star *}$ \\
\hline & $\mathrm{MO}\left(\times 10^{9} / \mathrm{L}\right)$ & $0.59 \pm 0.42$ & $0.29 \pm 0.07$ & $4.0431^{\star *}$ \\
\hline & PLT $\left(\times 10^{9} / \mathrm{L}\right)$ & $196.99 \pm 92.89$ & $237.63 \pm 37.98$ & $2.4546^{*}$ \\
\hline \multirow{6}{*}{$\begin{array}{l}\text { 2) D-D and } \\
\text { coagulation } \\
\text { indicators }\end{array}$} & PT (s) & $13.96 \pm 5.98$ & $11.16 \pm 8.17$ & 1.7863 \\
\hline & TT (s) & $17.93 \pm 3.83$ & $17.66 \pm 0.71$ & 0.5381 \\
\hline & $\operatorname{APTT}(\mathrm{s})$ & $31.46 \pm 13.55$ & $27.67 \pm 2.53$ & 1.9162 \\
\hline & $\mathrm{AT}(\%)$ & $85.88 \pm 18.41$ & $112.22 \pm 16.57$ & $2.9963^{\star *}$ \\
\hline & $\mathrm{FB}(\mathrm{g} / \mathrm{L})$ & $3.57 \pm 1.24$ & $2.71 \pm 0.52$ & $2.2013^{*}$ \\
\hline & INR & $1.19 \pm 0.52$ & $1.08 \pm 0.06$ & 0.8968 \\
\hline
\end{tabular}

${ }^{*} P<0.05,{ }^{* *} P<0.01$, the rest are all $>0.05$.

Table 4. Correlation between NLR, D-D, CAR and hematology, coagulation indicators.

\begin{tabular}{|c|c|c|c|c|c|c|}
\hline & \multicolumn{2}{|c|}{ CAR } & \multicolumn{2}{|c|}{ NLR } & \multicolumn{2}{|c|}{ D-D } \\
\hline & $r$ & $t$ & $r$ & $t$ & $r$ & $t$ \\
\hline $\mathrm{HB}$ & -0.2195 & $4.3039^{\star *}$ & -0.0851 & 1.6087 & -0.1237 & $2.3652^{\star}$ \\
\hline WBC & 0.3083 & $6.1660^{* *}$ & 0.5334 & $11.9574^{* *}$ & 0.1985 & $3.8395^{\star *}$ \\
\hline $\mathrm{NE}$ & 0.3576 & $7.9467^{\star \star}$ & 0.6894 & $18.0471^{\star *}$ & 0.1611 & $3.8395^{\star \star}$ \\
\hline LY & -0.1014 & 1.9500 & -0.4519 & 9.6354 & 0.0746 & 1.4102 \\
\hline MO & 0.1474 & $2.8292^{\star \star}$ & 0.0280 & 0.5323 & 0.2988 & $5.9405^{\star \star}$ \\
\hline PLT & 0.0953 & 1.7813 & -0.0128 & 0.2429 & -0.1127 & $2.1508^{*}$ \\
\hline $\mathrm{AT}$ & -0.2431 & $4.7667^{\star \star}$ & -0.1486 & $2.8577^{\star \star}$ & 0.0242 & 0.4575 \\
\hline FB & 0.6215 & $15.0484^{\star *}$ & 0.1864 & $3.5985^{\star *}$ & -0.0266 & 0.5028 \\
\hline
\end{tabular}

${ }^{\star} P<0.05,{ }^{* *} P<0.01$, the rest are all $>0.05$.

Table 5. Diagnostic value of NLR, D-D, and CAR respectively and in combination for PTE.

\begin{tabular}{ccccccc}
\hline Indicators & $\begin{array}{c}\text { Diagnostic } \\
\text { critical value }\end{array}$ & AUC & $95 \%$ CI & $\begin{array}{c}\text { Sensitivity } \\
(\%)\end{array}$ & $\begin{array}{c}\text { Specificity } \\
(\%)\end{array}$ & $P$ \\
\hline D-D & $3.89 \mathrm{mg} / \mathrm{L}$ & 0.669 & $0.601-0.725$ & 87.5 & 42.3 & $<0.05$ \\
CAR & 1.95 & 0.681 & $0.613-0.749$ & 62.5 & 71.5 & $<0.05$ \\
NLR & 7.54 & 0.495 & $0.577-0.725$ & 59.4 & 69.1 & $<0.05$ \\
D-D + NLR + CAR & Same as above & 0.704 & $0.638-0.770$ & 70.3 & 61.7 & $<0.05$ \\
\hline
\end{tabular}




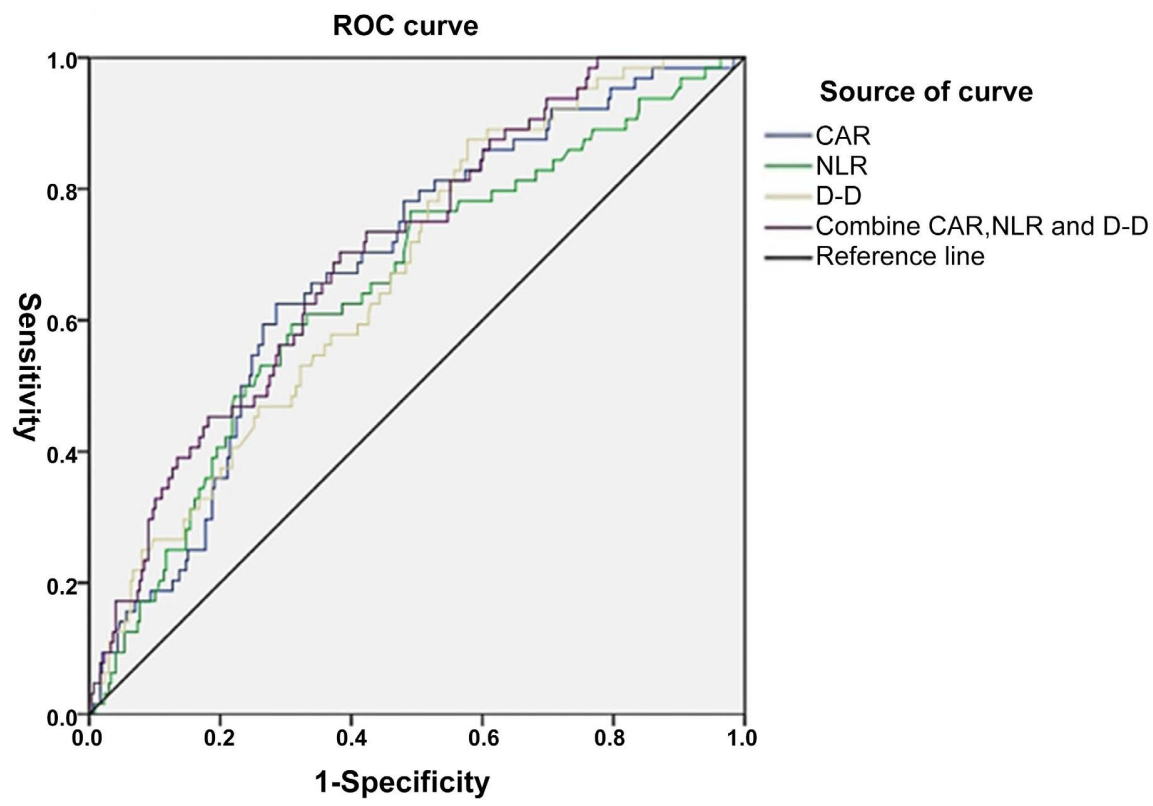

Figure 1. ROC curves about comparison of the diagnostic value of NLR, D-D, and CAR respectively and in combination for the PTE patients.

higher than that in the control group, which was consistent with the above literature results [9]. Although ROC curve showed that D-D had a diagnostic sensitivity of $87.5 \%$ to PTE, its specificity was very low (only $42.3 \%$ ). Therefore, it was necessary to explore new diagnostic indicators or combine several indicators to improve the specificity of diagnosis.

In this study, we found that inflammatory factors such as NLR, LMR, CRP, ALB, CAR, PLR and D-D were all significantly higher than those in the control group (Due to non-normal distribution, SII was not statistically significant, unless changed to rank-sum test). These results are consistent with some researches [10] [11], which indicating that PTE patients did have noteworthy inflammatory responses during the disease course. At the same time, correlation analysis found that the correlation degree of NLR, LMR and PLR among all inflammatory indicators was the highest, mainly because there were many repeated parameters in these three groups of indicators. In addition, considering that CRP and ALB had formed a new indicator (CAR), we selected NLR, D-D and CAR to conduct relevant studies on the diagnostic value of PTE.

The HB level of PTE patients was lower than that of the control group, which was consistent with our previous research results [12]. Inflammation can cause high expression of Hepcidin, which can lead to the failure of the body to use iron and cause anemia of patients. Although D-D was correlated with $\mathrm{HB}$ to a certain extent, it was obviously not enough to diagnose PTE. The levels of WBC, NE and MO were significantly higher than those of the control group, while LY was significantly lower than those of the control group, which also indicated that PTE patients had abnormal inflammatory response and immune function. Both NLR and CAR were significantly higher than those in the control group, which was 
consistent with the results reported in the literature [13] [14] [15]. NE releases inflammasome, a small molecule that participates in the formation of extracellular bactericidal substances of NE, releases protease and interacts with platelets to promote thrombosis [16], indicating that there is a close relationship between inflammation and thrombosis [5]. Kapoor and others [17] and Manfredi and others [4] all confirmed the close relationship between NLR and thrombotic diseases. This study showed that the sensitivity of NLR in diagnosing PTE was $59.4 \%$, but the specificity was significantly higher than D-D and close to CAR. This result was basically consistent with that of Wang Yang-bin and others [18]. Therefore, it is of certain value for the diagnosis of PTE to a certain extent.

As an accurate and reliable indicator of inflammation, CRP increases dramatically after tissue injury or inflammation, and it is produced by hepatocytes and epithelial cells under the stimulation of IL-6, IL-1, TNF and other inflammatory factors to remove pathogenic microorganisms and damaged, necrotic, apoptotic tissue cells that invade the body. PTE itself also can cause CRP level' increase through the induction of inflammation by thrombosis [1]. Recent studies [6] [8] [19] [20] have found that CRP is associated with the prognosis of solid tumors, and it has high specificity, especially when combined with nutritional indicator ALB. However, its diagnostic value in PTE has rarely been reported. Our research showed that its sensitivity was $62.5 \%$, lower than D-D, but slightly higher than NLR, and the specificity of CAR was the highest (71.5\%), which indicate that CAR has great auxiliary value for the clinical diagnosis and deserves further study.

ROC curve results showed that D-D had the highest sensitivity and the lowest specificity. Specificity was higher than sensitivity for both NLR and CAR. The combined detection of NLR, D-D and CAR showed the largest area under the AUC curve, and the sensitivity and specificity were significantly improved $(P<$ $0.05)$, suggesting that the combined detection of NLR, D-D and CAR is of great significance for the clinical diagnosis of PTE.

Köse $\mathrm{N}$ and others confirmed that age was also an independent prognosis factor for PTE patients [21]. However, in our study, elderly patients accounted for more and the average age of the elderly patients was 71.2 years, given that the small number of cases included in the study, we didn't analyze the factors of age in detail. In addition, the results may have some limitations for that this study was a single-center study and a retrospective analysis. Therefore, we will conduct more prospective studies, further expand the number of cases, unify the diagnostic criteria and parameter indexes, and strictly implement the inclusion and exclusion criteria of patients in a joint multi-center study, in order to further verify the value of NLR, D-D and CAR combined detection in the auxiliary diagnosis of PTE.

\section{Acknowledgements}

All authors read and approved the final manuscript. 


\section{Conflicts of Interest}

No potential conflict of interest was reported by the authors.

\section{References}

[1] Chirinos, J.A., Heresi, G.A., Velasquez, H., Jy, W., Jimenez, J.J., Ahn, E., et al. (2005) Elevation of Endothelial Microparticles, Platelets, and Leukocyte Activation in $\mathrm{Pa}$ tients with Venous Thromboembolism. Journal of the American College of Cardiology, 45, 1467-1471. https://doi.org/10.1016/j.jacc.2004.12.075

[2] Saghazadeh, A., Hafizi, S. and Rezaei, N. (2015) Inflammation in Venous Thromboembolism: Cause or Consequence? International Immunopharmacology, 28, 655. https://doi.org/10.1016/j.intimp.2015.07.044

[3] Zhang, J.Z. and Gu, J. (2016) Research Progress on Inflammasome and Cancer-associated Thrombosis-Review. Journal of Experimental Hematology, 24, 1260 1263.

[4] Manfredi, A.A., Ramirez, G.A., Rovere-Querini, P. and Maugeri, N. (2018) The Neutrophil's Choice: Phagocytose vs Make Neutrophil Extracellular Traps. Frontiers in Immunology, 9, Article No. 288. https://doi.org/10.3389/fimmu.2018.00288

[5] Gupta, N., Sahu, A., Prabhakar, A., Chatterjee, T., Tyagi, T., Kumari, B., et al. (2017) Activation of NLRP3 Inflammasome Complex Potentiates Venous Thrombosis in Response to Hypoxia. Proceedings of the National Academy of Sciences of the United States of America, 114, 4763-4768. https://doi.org/10.1073/pnas.1620458114

[6] Kinoshita, A., Onoda, H., Imai, N., Iwaku, A., Oishi, M., Tanaka, K., et al. (2015) The C-Reactive Protein/Albumin Ratio, a Novel Inflammation-Based Prognostic Score, Predicts Outcomes in Patients with Hepatocellular Carcinoma. Annals of Surgical Oncology, 22, 803-810. https://doi.org/10.1245/s10434-014-4048-0

[7] Wu, M., Guo, J., Guo, L. and Zuo, Q. (2016) The C-Reactive Protein/Albumin Ratio Predicts Overall Survival of Patients with Advanced Pancreatic Cancer. Tumor Biology, 37, 12525-12533. https://doi.org/10.1007/s13277-016-5122-y

[8] Zhou, T., Zhan, J., Hong, S., Hu, Z., Fang, W., Qin, T., et al. (2015) Ratio of C-Reactive Protein/Albumin Is an Inflammatory Prognostic Score for Predicting Overall Survival of Patients with Small-Cell Lung Cancer. Scientific Reports, 5, Article No. 10481. https://doi.org/10.1038/srep10481

[9] Li, W.J., Sha, M., Ma, W., Zhang, Z.P., Wu, Y.J. and Shi, D.M. (2016) Efficacy Evaluation of D-Dimer and Modified Criteria in Overt and Nonovert Disseminated Intravascular Coagulation Diagnosis. International Journal of Laboratory Hematology, 38, 151-159. https://doi.org/10.1111/ijlh.12467

[10] Wang, Q., Ma, J., Jiang, Z. and Ming, L. (2018) Prognostic Value of Neutrophil-to-Lymphocyte Ratio and Platelet-to-Lymphocyte Ratio in Acute Pulmonary Embolism: A Systematic Review and Meta-Analysis. International Angiology, 37, 4-11. https://doi.org/10.23736/S0392-9590.17.03848-2

[11] Bakirci, E.M., Topcu, S., Kalkan, K., Halil Tanboga, I., Borekci, A., Sevimli, S., et al. (2015) The Role of the Nonspecific Inflammatory Markers in Determining the Anatomic Extent of Venous Thromboembolism. Clinical and Applied Thrombosis/Hemostasis, 21, 181-185. https://doi.org/10.1177/1076029613494469

[12] Cheng, Z., Yan, M., Lu, Y. and Pan, X.-T. (2020) Expression of Serum BMP6 and Hepcidin in Cancer-Related Anemia. Hematology, 25, 134-138.

https://doi.org/10.1080/16078454.2020.1738098

[13] Wiwanitkit, V. (2016) Neutrophil to Lymphocyte Ratio in Allergic Rhinitis. Euro- 
pean Archives of Oto-Rhino-Laryngology, 273, 3443.

https://doi.org/10.1007/s00405-015-3886-0

[14] Lee, H., Um, S.J., Kim, Y.S., Kim, D.K., Jang, A.S., Choi, H.S., et al. (2016) Association of the Neutrophil-to-Lymphocyte Ratio with Lung Function and Exacerbations in Patients with Chronic Obstructive Pulmonary Disease. PLoS ONE, 11, Article ID: e0156511. https://doi.org/10.1371/journal.pone.0156511

[15] Kolber, W., Kuśnierz-Cabala, B., Maraj, M., Kielar, M., Mazur, P., Maziarz, B., et al. (2018) Neutrophil to Lymphocyte Ratio at the Early Phase of Acute Pancreatitis Correlates with Serum Urokinase-Type Plasminogen Activator Receptor and Interleukin 6 and Predicts Organ Failure. Folia Medica Cracoviensia, 58, 57-74.

[16] Petersen, E., Wågberg, F. and Angquist, K.A. (2002) Proteolysis of the Abdominal Aortic Aneurysm Wall and the Association with Rupture. European Journal of Vascular and Endovascular Surgery, 23, 153-157.

https://doi.org/10.1053/ejvs.2001.1572

[17] Kapoor, S., Opneja, A. and Nayak, L. (2018) The Role of Neutrophils in Thrombosis. Thrombosis Research, 170, 87-96. https://doi.org/10.1016/j.thromres.2018.08.005

[18] Wang, Y.B., Xia, M. and Xia, Y.Q. (2019) Plasma vWF, D-Dimer Levels Combined with Peripheral Blood Neutrophil/Lymphocyte Ratio to Detect Clinical Diagnostic Value in Thrombosis Disease. Journal of Modern Inspection Medicine, 34, 86-89.

[19] Joffe, L., Dwyer, S., Glade Bender, J.L., Lindsay Frazier, A. and Ladas, E.J. (2019) Nutritional Status and Clinical Outcomes in Pediatric Patients with Solid Tumors: A Systematic Review of the Literature. Seminars in Oncology, 46, 48-56. https://doi.org/10.1053/j.seminoncol.2018.11.005

[20] Bairey, O., Shacham-Abulafia, A., Shpilberg, O. and Gurion, R. (2016) Serum Albumin Level at Diagnosis of Diffuse Large B-Cell Lymphoma: An Important Simple Prognostic Factor. Hematological Oncology, 34, 184-192. https://doi.org/10.1002/hon.2233

[21] Köse, N., Yıldırım, T., Akın, F., Yıldırım, S.E. and İbrahim, A. (2020) Prognostic Role of NLR, PLR, and LMR in Patients with Pulmonary Embolism. Bosnian Journal of Basic Medical Sciences, 20, 248-253. https://doi.org/10.17305/bjbms.2019.4445 